\title{
ASO Visual Abstract: Short-Term Outcomes Following Breast Cancer Surgery With and Without Neoadjuvant Chemotherapy: A Nationwide Administrative Database Study in Japan
}

Takaaki Konishi, $\mathrm{MD}^{1,2}$ (D), Michimasa Fujiogi, $\mathrm{MD}^{2,3}$, Ayaka Sato, MD, PhD ${ }^{1}$, Nobuaki Michihata, MD, MPH, $\mathbf{P h D}^{4}$, Ryosuke Kumazawa, MPharm ${ }^{2}$, Hiroki Matsui, $\mathbf{M P H}^{2}$, Kiyohide Fushimi, MD, $\mathbf{P h D}^{5}$, Masahiko Tanabe, MD, $\mathrm{PhD}^{1}$, Yasuyuki Seto, $\mathrm{MD}, \mathrm{PhD}^{1}$, and Hideo Yasunaga, $\mathrm{MD}, \mathrm{PhD}^{2}$

${ }^{1}$ Department of Breast and Endocrine Surgery, Graduate School of Medicine, The University of Tokyo, Tokyo, Japan; ${ }^{2}$ Department of Clinical Epidemiology and Health Economics, School of Public Health, The University of Tokyo, Tokyo, Japan; ${ }^{3}$ Department of Emergency Medicine, Massachusetts General Hospital, Harvard Medical School, Boston, MA; ${ }^{4}$ Department of Health Services Research, Graduate School of Medicine, The University of Tokyo, Tokyo, Japan; ${ }^{5}$ Department of Health Policy and Informatics, Tokyo Medical and Dental University Graduate School, Tokyo, Japan

The association of neoadjuvant chemotherapy and chemotherapy-surgery interval with complications following breast cancer surgery remains unclear. Matchedpair cohort analyses revealed neoadjuvant chemotherapy was not associated with complications, regardless of interval. Patients can safely receive perioperative treatment without a lengthened interval (https://doi.org/10.1245/s10 434-021-11212-6).

Short-Term Outcomes Following Breast Cancer Surgery With and Without Neoadjuvant Chemotherapy: A Nationwide Administrative Database Study in Japan

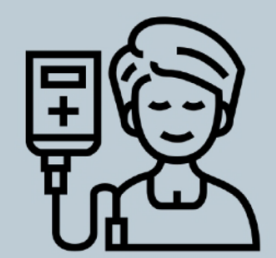

Neoadjuvant chemotherapy before breast cancer surgery

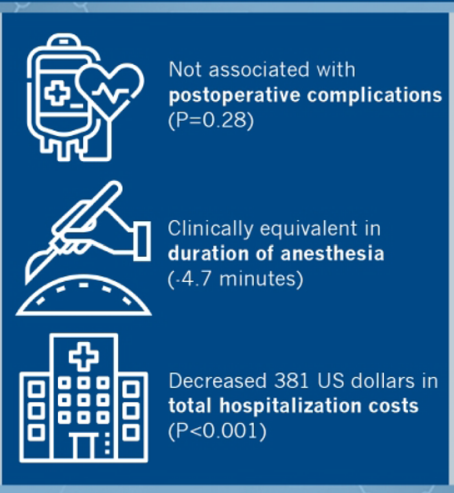

Konishi T, et al. Ann Surg Oncol.

Visual Abstract by @konishita_sur for @AnnSurgOncol
The results did not differ according to
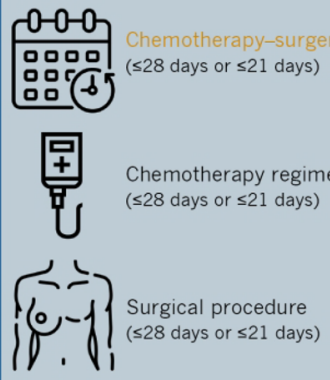

Chemotherapy regimen

( $\leqslant 28$ days or $\leqslant 21$ days)
( $\leq 28$ days or $\leq 21$ days)

\section{(5)}

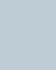

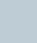

